\title{
Sphere-forming-like cells (squamospheres) with cancer stem-like cell traits from VX2 rabbit buccal squamous cell carcinoma
}

\begin{abstract}
Yuk-Kwan Chen ${ }^{1,2,3}$, Anderson Hsien-Cheng Huang ${ }^{1}$ and Li-Min Lin ${ }^{1,2,3}$
Previous studies have demonstrated that spheroid type cells grown under suspension culture conditions have cancer stem cell (CSC) traits in a number of cancers, but this phenomenon has not yet been reported in the VX2 rabbit oral cancer model. Hence, this study aimed to study the spheroid cells from VX2 rabbit buccal squamous cell carcinomas (SCCs) and assess their CSC characteristics. Five adult male New Zealand white outbred rabbits were used to generate VX2 rabbit buccal SCC. Sphere-forming cell culture was performed for the VX2 rabbit buccal SCC specimens. The self-renewal capability; cluster of designation (CD) 44, CD133, acetaldehyde dehydrogenase 1 (ALDH1), B cell-specific Moloney murine leukemia virus integration site 1 (Bmi-1), Nestin, octamer-binding transcription factor 4 (Oct4) and reduced expression protein-1 (Rex-1) expression with reverse transcription-polymerase chain reaction (RT-PCR); chemoresistance to cisplatin and 5-fluorouracil; and in vivo tumorigenicity of spheroid cell transplantation in nude mice were evaluated to determine the CSC characteristics of the resulting spheroid cells. We successfully obtained spheroid cells from the VX2 rabbit OSCC tissues. The spheroid cells exhibited CSC traits, including the expression of CSC and stem cell markers (CD44, Bmi-1, Nestin, Oct4 and Rex-1), capacity to generate new spheroid colonies within 1 week of reseeding from single-dissociated spheroid cells, chemoresistance capacity and generation of tumour xenografts (with histological features resembling those of the original VX2 rabbit buccal SCC) from the transplantation of $10^{3}$ undifferentiated spheroid cells into nude mice. In summary, we demonstrated that spheroid cells with CSC cell traits can be derived from VX2 rabbit buccal SCCs, indicating that this animal cancer model is applicable for studying CSCs in human oral cancers.
\end{abstract} International Journal of Oral Science (2014) 6, 212-218; doi:10.1038/ijos.2014.32; published 11 July 2014

Keywords: cancer stem-like cell; squamosphere; VX2 rabbit oral carcinoma

\section{INTRODUCTION}

Oral squamous cell carcinoma (OSCC) accounts for more than $90 \%$ of oral malignancies and is the eleventh most common global cancer, comprising $3 \%$ of all newly diagnosed cancer cases. ${ }^{1-2}$ Due to the high prevalence of chewing betel-quid and/or tobacco in Central and Southeast Asian countries and regions including Taiwan, OSCC is one of the leading cancer types and the fourth most common cancer in males as well as the fifth leading cause of cancer death in Taiwanese males. ${ }^{3-4}$ Today, the standard care for patients with OSCC consists of surgery, chemotherapy and radiotherapy, but the survival of OSCC patients has not significantly improved. ${ }^{3}$ Continued study is needed to explore the mechanism for the initial stages of oral carcinogenesis. Cancer stem cells (CSCs) are of great interest in the study of the pathogenesis of oral cancer. CSCs are tumorigenic, multipotential cells with poorly regulated self-renewal characteristics. ${ }^{5}$ Accumulating evidence suggests that OSCC CSCs play an important role in the initiation, maintenance, growth and metastasis of tumours. ${ }^{6-8}$

Selection of an appropriate preclinical animal cancer model is useful for elucidating the molecular pathogenesis of OSCC. The VX2 cell line has been applied in many carcinoma studies, including OSCC research. ${ }^{9}$ We established a novel application in which VX2 cancer cells are transplanted into the buccal mucosa of rabbits to induce buccal mucosa SCC. ${ }^{10}$

Previous studies have demonstrated that cells that grow as a spheroid type in suspension culture conditions display stem cell properties in a number of cancers. ${ }^{11-12}$ To the best of our knowledge, no similar studies have reported the use of a VX2 rabbit oral cancer model. In the current study, we aimed to evaluate sphere-forming-like cells (squamospheres) from VX2 rabbit buccal SCC using suspension culture conditions; we also investigated their CSC characteristics.

\section{MATERIALS AND METHODS}

Generation of VX2 rabbit buccal SCC

The experimental protocol was approved by the Animal Experiments Committee at our institution to ensure humane treatment of the test animals; all procedures were implemented in adherence with the National Institute of Health guide for the care and use of animals. Five adult male, specific-pathogen free, New Zealand White outbred

${ }^{1}$ Department of Oral Pathology, Faculty of Dentistry, College of Dental Medicine, Kaohsiung Medical University, Kaohsiung, Taiwan; ${ }^{2}$ Division of Dentistry, Department of Oral

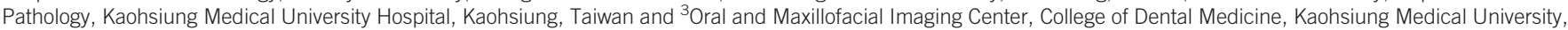
Kaohsiung, Taiwan

Correspondence: Dr LM Lin, Department of Oral Pathology, Faculty of Dentistry, College of Dental Medicine, Kaohsiung Medical University, Kaohsiung 807, Taiwan E-mail: k0285@ms22.hinet.net

Accepted 28 April 2014 
rabbits weighing 2.5-3.5 kg (purchased from the Livestock Research Institute, Council of Agriculture, Executive Yuan, Taiwan) were used for generating the VX2 rabbit buccal SCC; our laboratory previously reported on the procedures. ${ }^{5}$ The VX2 tumour tissues were excised, fixed in $10 \%$ buffered formalin and embedded in paraffin for haematoxylin-eosin staining.

\section{Sphere-forming cell culture for VX2 rabbit buccal SCC specimens}

The procedures for the sphere-forming cell culture conditions were as previously reported; we implemented some modifications. ${ }^{13}$ The VX2 rabbit buccal SCC specimens were minced into tiny pieces with aseptic surgical blades. These pieces were enzymatically digested using collagenase (Gibco, Carlsbad, CA, USA), hyaluronidase and DNase (Sigma-Aldrich, St Louis, MO, USA), and they were then incubated for $2 \mathrm{~h}$ at $37{ }^{\circ} \mathrm{C}$ with $5 \% \mathrm{CO}_{2}$. After pipetting every $15 \mathrm{~min}$ with a sterilized $10-\mathrm{mL}$ pipette, the resulting cell disaggregates were washed twice with phosphate-buffered saline and subsequently centrifuged with Percoll (Sigma-Aldrich, St Louis, MO, USA) to remove any cellular debris and contaminants. The cell suspension was filtered through a $40-\mu \mathrm{m}$ cell strainer. The resulting single cells were placed in serum-free medium (Dulbecco modified Eagle medium, DMEM/F12) supplemented with $20 \mathrm{ng} \cdot \mathrm{mL}^{-1}$ of basic fibroblast growth factor and epidermal growth factor (PeproTech, Rocky Hill, NJ, USA), $5 \mu \mathrm{g} \cdot \mathrm{mL}^{-1}$ of insulin (Sigma-Aldrich, St Louis, MO, USA), $0.4 \%$ bovine serum albumin and $0.02 \times \mathrm{B} 27$ (Invitrogen, Carlsbad, CA, USA). The culture medium was replaced or supplemented with additional epidermal growth factor and basic epidermal growth factor every 3 days. When the sphere-forminglike cells in the suspension culture attained a diameter of more than $20 \mu \mathrm{m}$, the cells were collected by mild centrifugation, dissociated into a single-cell suspension with the aforementioned enzymes, and then cultured using the aforementioned stem cell suspension culture conditions to promote the generation of sphere-forming-like cells. Third-generation sphere-forming-like cells were used in the following experiments.

\section{Reverse transcription-polymerase chain reaction}

Reverse transcription (RT)-polymerase chain reaction (PCR) was performed using a DNA thermal cycler (TaKaRa MP, Tokyo, Japan). The total RNA samples were prepared from sphere-forming-like cells using TRIzol reagent (Invitrogen, Carlsbad, CA, USA). Reverse transcription was performed from $500 \mathrm{ng}$ of total RNA template using a High Capacity cDNA Reverse Transcription Kit (Applied Biosystems, Foster City, CA, USA). The resulting complementary DNA sample was subsequently used for PCR amplification. The PCR reactions were performed using specific oligoprimer sets, which were designed according to the published complementary DNA sequences. All oligoprimers were designed across the junction between two exons; the forward primer was located at the $3^{\prime}$ region of the leading exon and the reverse primer was located at the $5^{\prime}$ region of the following exon to minimize the possibility of a false-positive or contamination from genomic DNA. We used the following sequences of the rabbit-specific primers:

Cluster of designation 44 (CD44) primers: forward 5' -ATCCACCCCAACTCCATCTGT-3', reverse 5'-TTGAGTGGAGCTGAGGCATTG-3'; CD133 primers: forward 5'-ACCAGCGGGAGAAAGAGGAC-3', reverse 5'-GTCCAAGAAGGTCCGCAGGT-3'; aldehyde dehydrogenase 1 (ALDH1) primers: forward 5'-GGCAGACTTACCTACCCCAC-3', reverse 5'-CCTGCCTTGCTGCCTTCACA-3'; B cell-specific Moloney murine leukemia virus integration site $1(B m i-1)$ primers: forward $5^{\prime}$ AAATCTAAGGAGGAGGTGAATGA-3', reverse 5' -CAATCTGGAAAGTGTTAGGTATG-3'; Nestin primers: forward 5'-AATTCCCGGCTTCAGACACCT-3', reverse 5'-CTCCAGGGTATCGGGCAAAG-3'; octamerbinding transcription factor 4 (Oct4) primers: forward $5^{\prime}$-CGGAAGAGAAAGCGAACGAGT-3', reverse 5' - CAGAACCACACACGGACCAC-3'; reduced expression protein-1 (Rex-1) primers: forward 5' CGGAAGGGCTCTGGTGGAAA-3' , reverse 5' -TGGTCAGTCTCACAGGGCAC-3'.

The amplification cycle consisted of $5 \mathrm{~min}$ at $94{ }^{\circ} \mathrm{C}, 30 \mathrm{~s}$ at $94{ }^{\circ} \mathrm{C}$, $30 \mathrm{~s}$ at $62{ }^{\circ} \mathrm{C}$ and $20 \mathrm{~s}$ at $72{ }^{\circ} \mathrm{C}$, which was repeated 40 times and then followed by $5 \mathrm{~min}$ at $72{ }^{\circ} \mathrm{C}$. In addition, DNase I-treated RNA (without RT) and $\mathrm{H}_{2} \mathrm{O}$ were used as controls. The amplification products were analysed by electrophoresis in a $2 \%$ agarose gel along with the relevant DNA molecular weight marker (Boehringer, Mannheim, Germany) and were then stained with ethidium bromide. The PCR products were visualized as bands using an ultraviolet transilluminator, and the expression levels were subsequently measured.

\section{Chemoagent sensitivity assay}

Sphere-forming-like cells were dissociated into single cells and then plated in a 96-well plate at a density of $7 \times 10^{3}$ cells per well. The sphereforming-like cells were treated with cisplatin $\left(10 \mu \mathrm{mmol} \cdot \mathrm{L}^{-1}\right)$ and 5-fluorouracil (5-FU) $\left(20 \mu \mathrm{mmol} \cdot \mathrm{L}^{-1}\right)$ and were then cultured at $37{ }^{\circ} \mathrm{C}$ and $5 \% \mathrm{CO}_{2}$. Twenty-four hours later, $20 \mu \mathrm{L}$ of 3-(4,4-dimethylthiazol2-yl)-2,5-diphenyltetrazolium bromide solution $\left(5 \mathrm{mg} \cdot \mathrm{L}^{-1}\right.$ in phosphate buffer solution) was added to each well and placed at room temperature for $3 \mathrm{~h}$. Finally, the absorbance was measured at a wavelength of $570 \mathrm{~nm}$.

\section{In vivo tumorigenicity analysis}

Six immunodeficient nude mice (BALB/c nu/nu male; 6 weeks old; weight, 20-22 g) were housed under specific pathogen-free conditions with a regular light/dark cycle and were allowed one week of adaptation to their new surroundings. They were fed standard rodent chow (Laboratory Rodent Diet 5001; Lab Diet, St Louis, MO, USA) with ad libitum access.

Different levels of sphere-forming-like cells were subcutaneously transplanted into the backs of the mice using a 22-gauge needle; two mice each were implanted with $10^{2}, 10^{3}$ and $10^{4}$ cells, respectively. Tumour growth was inspected visually and palpated weekly to monitor tumour formation 7 weeks post-transplantation. The mice were then euthanized by cervical decapitation and the induced tumour tissues were excised, fixed in $10 \%$ buffered formalin and embedded in paraffin for subsequent haematoxylin-eosin staining. The histological characteristics of the tumour xenografts were compared with the original VX2 rabbit buccal tumour.

\section{Analyses of the first-generation sphere-forming-like cells}

Western blot. Total proteins were extracted and concentrated for analysis of the first-generation sphere-forming-like cells using the Bradford assay (Bio-Rad, Hercules, CA, USA); total proteins were also extracted from the normal rabbit buccal tissues (used as the control). Equal levels of the protein were boiled prior to polyacrylamide gel electrophoresis; the proteins were transferred onto a polyvinylidene difluoride membrane (cat. no. IPVH 00010, Millipore Immobilon P; Millipore, Billerica, MA, USA) using Bio-Rad's Transblot. The membrane was then blocked, treated with primary antibodies (CD-44: cat. no. SC-7297; Santa Cruz Biotechnology, Santa Cruz, CA, USA with 1:2 000. Bmi-1: cat. no. GTX114008; Rex-1: cat. no. GTX101903; Oct4: cat. no. GTX101497; Nestin: cat. no. GTX116066, Gene Tex, Irvine, CA, USA; each with $1: 2000)$ and $\gamma$-tubulin $(1: 2000$; cat. no. T6557; Sigma-Aldrich, St Louis, MO, USA), followed secondary antibody, 
and then detected using an Amersham's ECL kit (Amersham, Pittsburgh, PA, USA). The relative expression levels upon Western blot analyses were measured and normalized to the expression level of the positive control.

Chemoagent sensitivity assay. With procedures similar to those described above, the chemoresistance of the first-generation sphereforming-like cells to cisplatin and 5-FU were evaluated.

In vivo tumorigenicity analysis. With procedures similar to those described above, different levels of the first-generation sphere-forming-like cells were subcutaneously transplanted into the backs of mice using a 22-gauge needle; two mice each were implanted with $10^{2}, 10^{3}$ and $10^{4}$ cells, respectively. Seven weeks post-transplantation, the mice were euthanized and the induced tumour tissues were excised, fixed in $10 \%$ buffered-formalin and embedded in paraffin for subsequent haematoxylin-eosin staining.

\section{Analyses of the primary culture cells from the VX2-induced carcinomas}

Western blot. The total proteins were extracted and concentrated for analysis of the primary culture cells from the VX2-induced carcinomas using the Bradford assay (Bio-Rad, Hercules, CA, USA); the same procedure was performed for the normal rabbit buccal tissues (used as the control). Equal levels of the protein were boiled prior to polyacrylamide gel electrophoresis; the proteins were transferred onto a polyvinylidene difluoride membrane (cat. no. IPVH 00010, Millipore Immobilon P) using Bio-Rad's Transblot. The membrane was then blocked, treated with primary antibodies (CD-44: cat. no. SC-7297; Bmi-1: cat. no. GTX114008) and $\gamma$-tubulin (1:2 000; cat. no. T6557), followed by secondary antibody, and finally detected using an Amersham's ECL kit. The relative expression levels were measured and normalized to the expression level of the positive control.

In vivo tumorigenicity analysis. With procedures similar to those described above, different levels of the primary culture cells from the VX2-induced carcinomas were subcutaneously transplanted into the backs of the mice using a 22-gauge needle; two mice each were implanted with $10^{2}, 10^{3}$ and $10^{4}$ cells, respectively. After implantation, each animal was observed daily for up to 7 weeks post-transplantation.

\section{RESULTS}

Moderately-differentiated VX2 buccal SCCs were found in all the rabbits (Figure 1), which is similar to our previous findings. ${ }^{5}$ We then further attempted to utilize stem cell suspension culture conditions to

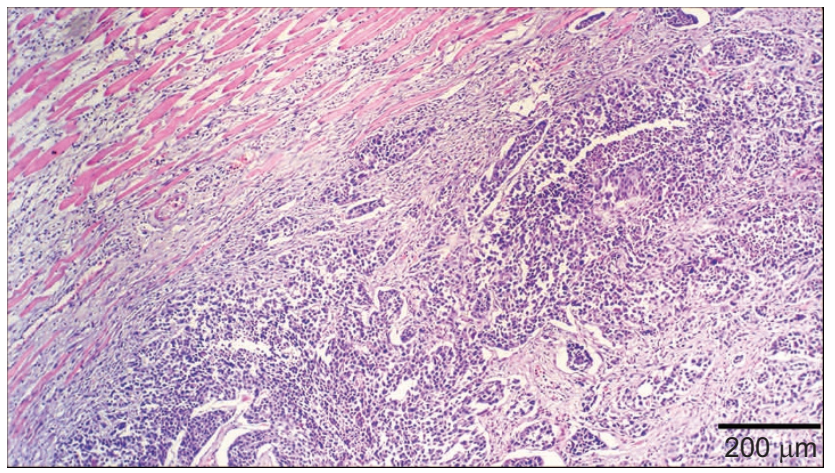

Figure 1 Histology of the VX2 rabbit buccal tumour. Moderately-differentiated VX2 rabbit buccal squamous cell carcinoma is noted (haematoxylin-eosin stain).

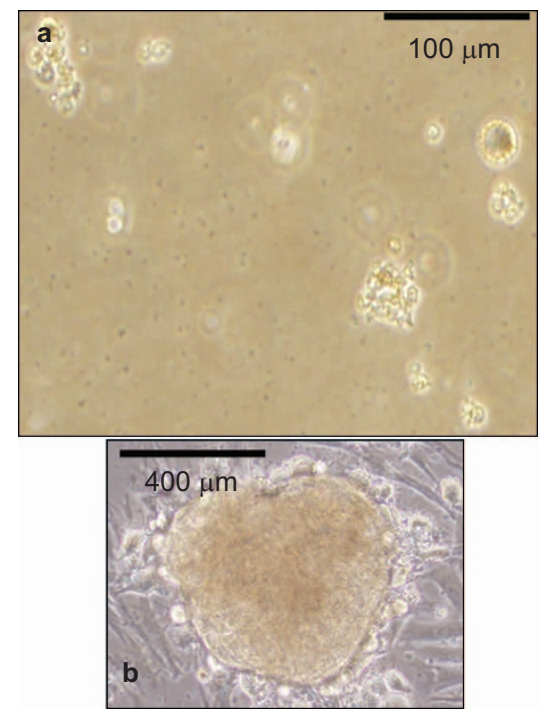

Figure 2 Sphere-forming cell cultures of VX2 rabbit buccal carcinoma specimens. Stem cell suspension culture conditions produced sphere-forming-like cells using single-dissociated cancer cells derived from VX2 buccal carcinoma specimens. Most of the produced sphere-forming-like cells exhibit morphology of a well-defined circular shape.

produce sphere-forming-like cells with single-dissociated cancer cells derived from the VX2 buccal SCCs, and clusters of sphere-forming cells were successfully generated after 2 weeks of culturing (Figure 2). Most of these sphere-forming-like cells had a distinct morphological appearance with a well-defined circular shape (Figure 2). Self-renewal capacity is an essential criterion for stem cells, and we found that the enzymatically-dissociated sphere-forming-like cells maintained their spheroid morphology during serial passaging, and the number of sphere-forming-like cells increased in the second and the third generations in comparison with the first generation (Figure 3). No statistically significant difference was noted for the comparison between the first and second generations ( $P>0.05$, Student's $t$-test); however, there were significant differences when the first and second generations, respectively, were compared with the third generation $(P<0.05$, Student's $t$-test).

Subsequently, we performed RT-PCR to examine whether the sphere-forming-like cells expressed cancer stem cell (CD44, CD133, ALDH1 and Bmi-1), stem cell (Oct4 and Rex-1) and differentiation

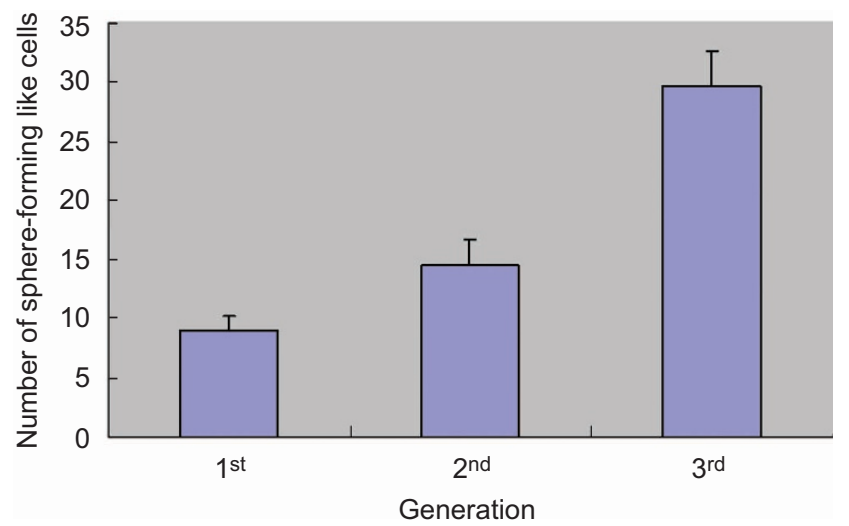

Figure 3 Self-renewal capacities of sphere-forming-like cells from VX2 buccal carcinoma. The single dissociated sphere-forming-like cells maintained a spheroid morphology following serial passaging for three consecutive generations. 

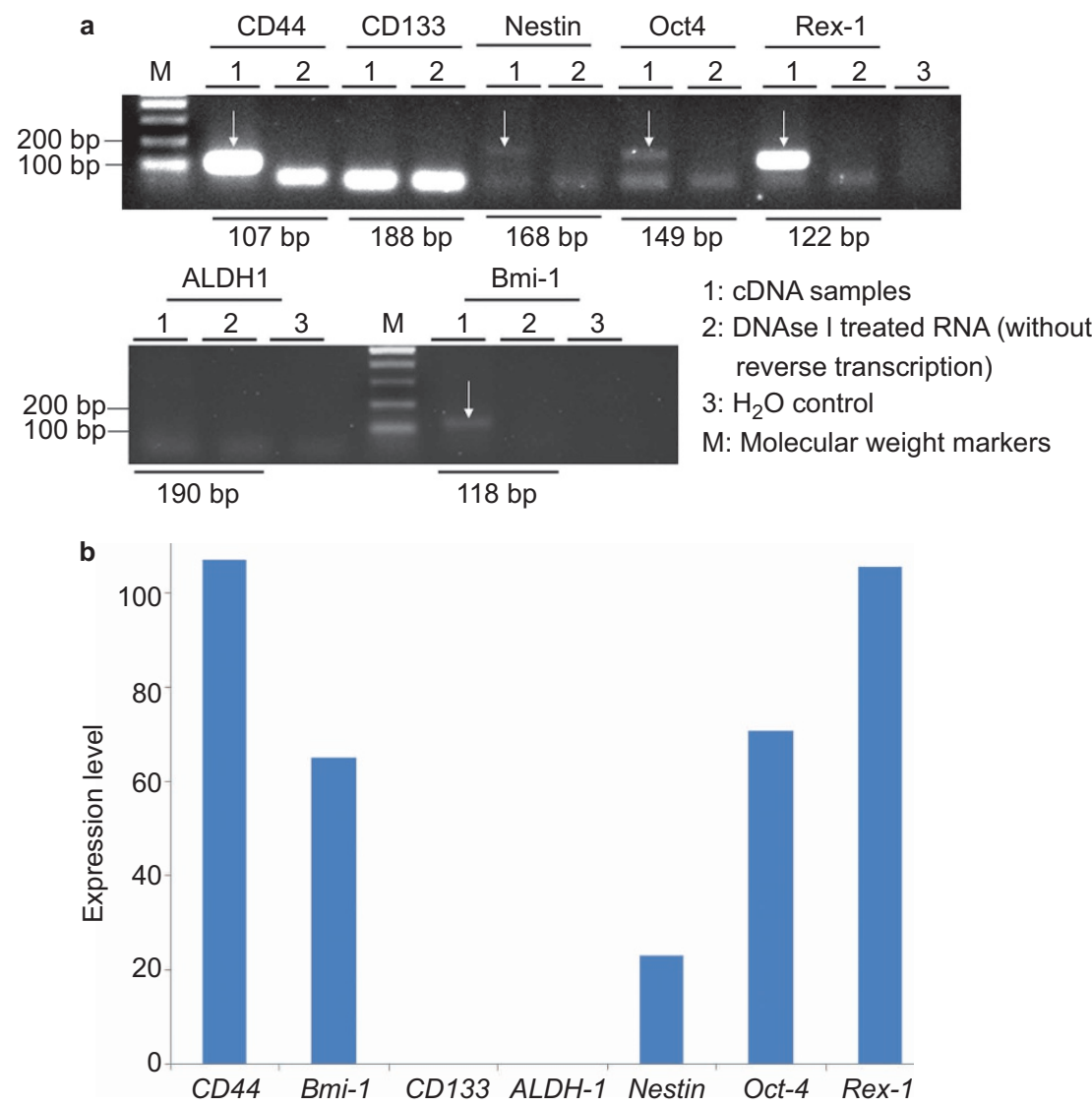

Figure 4 Reverse transcription-polymerase chain reaction. Messenger RNA (mRNA) expression of both CD44 and Bmi- 1 (white arrows) was observed in the sphereforming-like cells from the VX2 buccal carcinoma, but CD133 and ALDH1 were not expressed; mRNA expression of Nestin, Oct4 and Rex-1 was also observed (white arrows).

(Nestin) markers. We observed mRNA expression of both CD44 and Bmi-1 in the sphere-forming-like cells but not of CD133 and ALDH1; the mRNA expression of Nestin, Oct4 and Rex-1 was also observed (Figure 4).

Additionally, we evaluated the chemoresistance of the sphereforming-like cells to cisplatin and 5-FU, chemotherapeutic agents. We found that these sphere-forming-like cells were resistant to the chemotherapeutic agents (Figure 5).

To further determine the tumorigenicity of the sphere-forming-like cells derived from VX2 rabbit buccal SCC in vivo, we subcutaneously injected varying numbers of sphere-forming-like cells $\left(10^{2}, 10^{3}\right.$ or $\left.10^{4}\right)$ into a total of six immunodeficient nude mice. We noted that the concentration of the $10^{3}$ cells was sufficient for producing visible tumours 7 weeks after transplantation of the sphere-forming-like cells (Figure 6a and 6b). Furthermore, the histological characteristics of the tumours that developed in the nude mice resembled those of the original VX2 rabbit buccal SCC (Figure 6c).

Although, as described above, the squamosphere formation capacity was increased by repeating the passage process (Figure 3 ), we further investigated the expression of cancer stem cell, stem cell and differentiation markers as well as the chemoresistance and in vivo tumorigenicity of the first-generation sphere-forming-like cells. First, using Western blot analysis, we found that the first-generation sphere-forming-like cells, like the $3^{\text {rd }}$-generation cells, expressed cancer stem cell (CD44 and Bmi-1), stem cell (Oct4 and Rex-1) and differentiation (Nestin) markers (Figure 7). Second, the $1^{\text {st }}$-generation sphere-forming-like cells demonstrated a lower chemoresistance capacity than the $3^{\text {rd }}$ generation cells (Figure 5), but these differences were not significant $(P>0.05$, Student's $t$-test $)$. Third, the in vivo tumorigenicity of the first-generation sphere-forming-like cells was compatible with that of the third-generation cells (Figure 8).

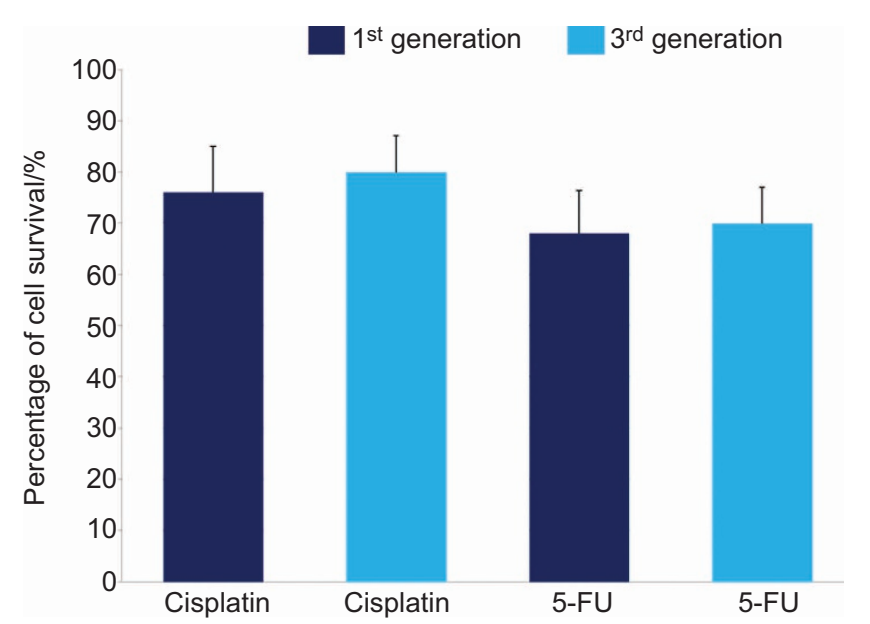

Figure 5 Chemoagent sensitivity assay. The percentage of cell survival of the third-passage sphere-forming-like-cells was slightly higher than that of the firstpassage sphere-forming-like-cells in the presence of cisplatin and 5-FU. 5-FU, 5fluorouracil. 

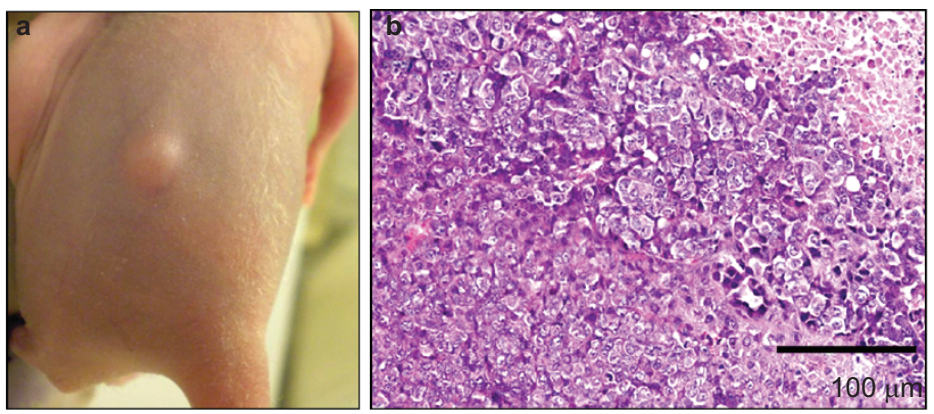

\begin{tabular}{|c|c|}
\hline $\begin{array}{l}\text { Concentrations of transplanted } \\
\text { sphere-forming like cells }\end{array}$ & $\begin{array}{l}\text { Nude mice with } \\
\text { tumors formation }\end{array}$ \\
\hline $10^{2}$ & $0 / 2$ \\
\hline $10^{3}$ & $1 / 2$ \\
\hline $10^{4}$ & $2 / 2$ \\
\hline $\begin{array}{l}\text { Concentrations of transplanted } 1^{\circ} \\
\text { culture cells of buccal carcinoma }\end{array}$ & $\begin{array}{l}\text { Nude mice with } \\
\text { tumors formation }\end{array}$ \\
\hline $10^{2}$ & $0 / 2$ \\
\hline $10^{3}$ & $0 / 2$ \\
\hline $10^{4}$ & $0 / 2$ \\
\hline
\end{tabular}

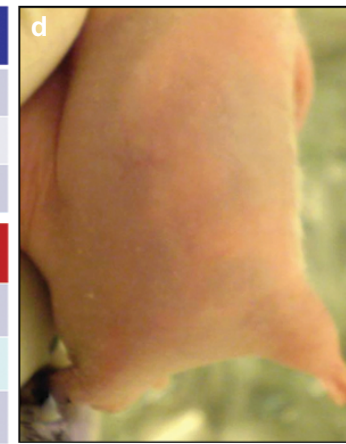

Figure 6 In vivo tumorigenesis assay. Varying numbers of sphere-forming-like cells from the VX2 rabbit buccal carcinoma were subcutaneously injected into nude mice. Representative image showing tumour xenograft formation (a) and representative micrograph showing the histological differentiation of the tumour xenograft (b) haematoxylin-eosin stain), which was similar to the original VX2 rabbit buccal carcinoma. As few as $10^{3}$ cells produced visible tumour formation within 7 weeks posttransplantation (c). Different numbers of primary culture cells from the VX2 rabbit buccal carcinoma were subcutaneously injected into nude mice. Representative image showing a lack of visible tumour xenograft formation (d) for cell concentrations up to $10^{4}$ (e).

a
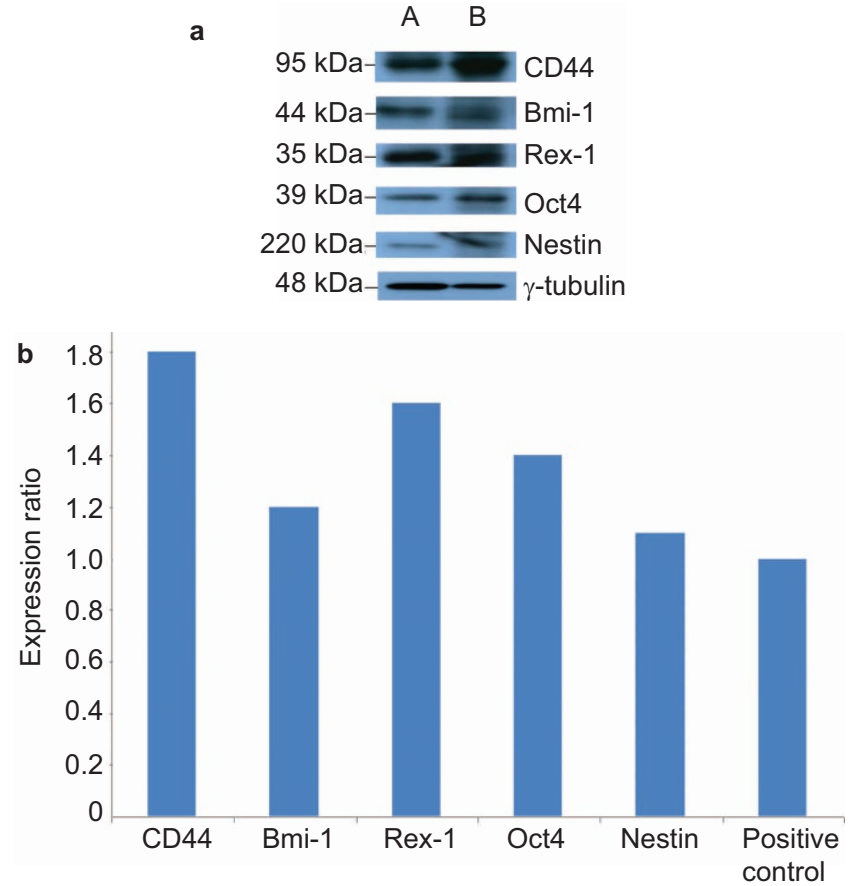

Figure 7 Western blot analyses of the first-passage sphere-forming-like cells. The first-passage sphere-forming-like cells from the VX2 rabbit buccal carcinoma expressed cancer stem cell (CD44 and Bmi-1), stem cell (Oct4 and Rex-1) and differentiation (Nestin) markers. lane A: positive control; lane B: first-passage sphere-forming-like cells.
Finally, we found that the primary culture cells from the VX2-induced carcinoma also expressed CD44 and Bmi-1, which is similar to the sphere-forming-like cells (Figure 9). Moreover, with transplantation of various numbers of the primary culture cells from the VX2-induced carcinoma into nude mice, no visible tumour formation was noted at

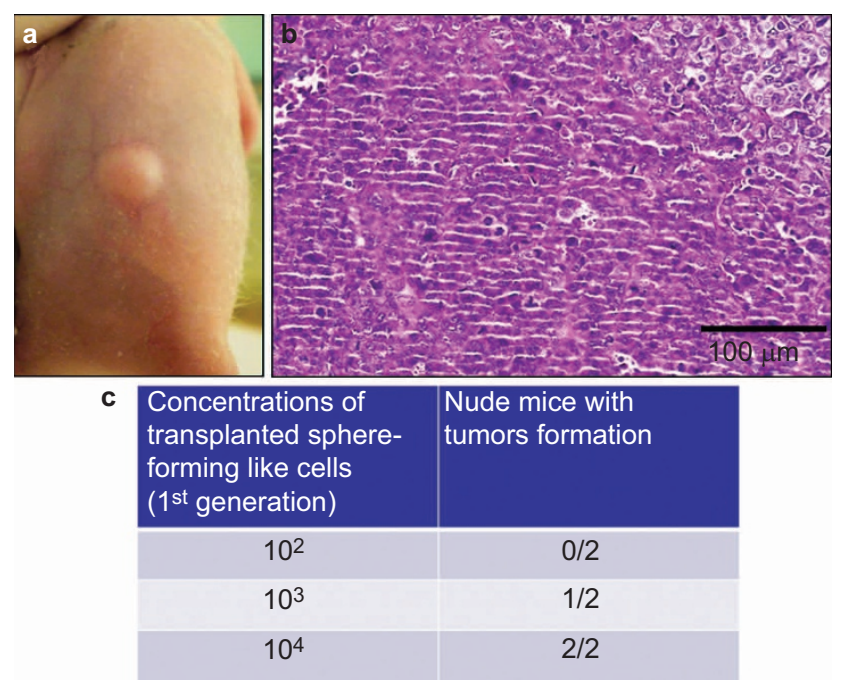

Figure 8 In vivo tumorigenesis assay of the first-passage sphere-forming-like cells. Different numbers of the first-passage sphere-forming-like cells from the VX2 rabbit buccal carcinoma were subcutaneously injected into nude mice. Representative image showing tumour xenograft formation (a) and representative micrograph showing the histological differentiation of the tumour xenograft (b, haematoxylin-eosin stain); $10^{3}$ cells could produce visible tumour formation within 7 weeks post-transplantation (c). 
a
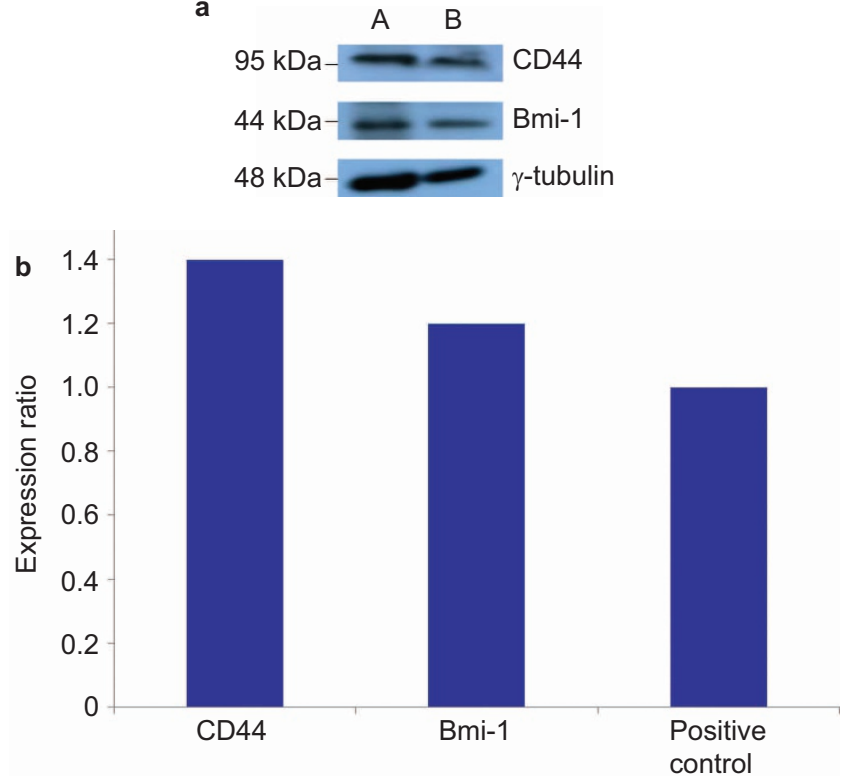

Figure 9 Western blot analyses of the primary culture cells from the VX2 rabbit buccal carcinoma. The primary culture cells from the VX2 rabbit buccal carcinoma expressed cancer stem cell (CD44 and Bmi-1) markers. lane A: primary culture cells from the VX2 rabbit buccal carcinoma; lane B: positive control.

a concentration of up to $10^{4}$ cells (Figure 6d and 6e); however, visible tumours were obtained for $10^{6}$ cells (unpublished data).

\section{DISCUSSION}

Various types of malignancies have a subpopulation of CSCs, but little is known regarding the CSCs and CSC surface markers in the VX2 rabbit oral cancer model. In the current study, we demonstrated that VX2 rabbit buccal SCC grown with sphere-forming cell suspension culture conditions can form tumour spheroids. These findings are in agreement with previous reports of other human malignancies, including brain, ovarian and lung cancer. ${ }^{1-13}$ Additionally, our findings are in agreement Lim et al., ${ }^{14}$ who reported on the CSC traits in squamospheres derived from primary head and neck SCCs, including OSCCs, suggesting that this animal cancer model may be applicable to future CSC study in human oral cancers.

Stem cells in spheroids undergo both symmetric and asymmetric division; symmetric division occurs in one stem cell, generating two daughter stem cells, while asymmetric division occurs in a daughter stem cell and differentiated daughter cell. The spheroid cells may consist of stem cells, progenitor cells and differentiated cells. Serial passaging of spheroid cells results in the exclusion of the more differentiated cells in favour of self-renewing stem cells that allow cancer cell sphere culturing to act as an in vitro model, which maximally maintains CSCs in an undifferentiated state. ${ }^{15-16}$ Therefore, although we found that expression of cancer stem cell, stem cell and differentiation markers as well as the chemoresistance and in vivo tumorigenicity of the $1^{\text {st }}$-passage sphere-forming-like cells were compatible with the third-passage sphere-forming-like cells derived from VX2 rabbit SCCs, we used the third-passage sphere-forming-like in our study.

CD44, a hyaluronate acid receptor, is a family of cell surface glycoproteins that participates in the cell adhesion, migration and metastasis of cancer cells; CD44 is also regarded as a CSC marker in head and neck SCC, including oral cavity counterparts. ${ }^{1-19}$ ALDH1, an isoform of aldehyde dehydrogenase (ALDH), is a cytosolic detoxifying isoenzyme that oxidizes intracellular aldehydes and leads to the oxidation of retinol to retinoic acid in early stem cell differentiation. ${ }^{20-21}$ ALDH1 has been reported to recognize CSCs in head and neck SCCs. ${ }^{22-23}$ CD133 is a glycosylated protein with five transmembrane domains and two large extracellular loops. ${ }^{24-25} \mathrm{CD} 133^{+}$stem-like cells in human OSCC possess a higher clonogenicity, invasiveness, and tumorigenesis than CD133 cells. ${ }^{26} \mathrm{Bmi}-1$, a stem cell-related gene, is a polycomb group transcription repressor that mediates gene silencing by regulating the chromatin structure, ${ }^{27}$ and Bmi- 1 participates

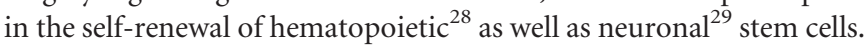
Bmi-1 is a marker for head and neck CSCs that plays a role in supporting the self-renewal characteristics and tumorigenicity of head neck SCC-derived CSCs. ${ }^{30}$ We found that both the sphere-forming-like cells and the primary culture cells from VX2 rabbit OSCC express CD44 and Bmi-1. In contrast, no CD133 or ALDH1 expression was noted. Hence, our results are partially in agreement with the aforementioned reports on human head and neck SCC.

Researchers generally use molecular markers to identify CSCs. In general, these markers are not unique to CSCs. Hence, the present tendency is to recruit multiple markers to improve specificity. In addition, there is increased evidence that the most suitable grouping of CSC markers may be tumour type-dependent. ${ }^{31}$ It has been suggested that the $\mathrm{CD} 44^{+} / \mathrm{Bmi}^{-} 1^{+} / \mathrm{CD} 133^{-} / \mathrm{ALDH}^{-}$status in the current study could be putatively combined as CSC markers for VX2-induced rabbit OSCC. However, to confirm this assumption, further experiments need to be performed by isolating the $\mathrm{CD} 44^{+} / \mathrm{Bmi}^{-} 1^{+} /$ $\mathrm{CD}_{133^{-}} / \mathrm{ALDH}^{-}$cell populations and implementing subsequent suspension culture to evaluate the levels of sphere-forming-like cells (squamospheres).

In general, there are several criteria of CSCs, including self-renewal, stem cell marker expression, chemoresistance, and tumour-initiating potential. ${ }^{32}$ We found that sphere-forming-like cells from VX2 rabbit OSCC have these CSC traits, wherein (i) the derived spheroid cells expressed a number of stem cell markers including CD44, Bmi1, Nestin, Oct4, and Rex-1; (ii) single-dissociated spheroid cells were capable of generating new spheroid clusters within one week of reseeding; (iii) the cells had chemoresistance to chemotherapeutic agents; and (iv) transplantation of as few as $10^{3}$ undifferentiated spheroid cells into immunodeficient nude mice produced tumour xenografts.

In summary, we have established spheroid cells from VX2 rabbit OSCC that possess the general traits of CSCs, including self-renewal, stem cell marker expression, chemoresistance and tumour-initiating potential. Our results imply that this animal cancer model may be applicable to future CSC studies of human oral cancer, especially for buccal SCC.

1 Parkin DM, Bray F, Ferlay J et al. Global cancer statistics, 2002. CA Cancer J Clin 2005; 55(2): 74-108.

2 Parkin DM, Pisani P, Ferlay J. Estimates of the worldwide incidence of 25 major cancers in 1990. Int J Cancer 1999; 80(6): 827-841.

3 Warnakulasuriya S. Global epidemiology of oral and oropharyngeal cancer. Oral Oncol 2009; 45(4/5): 309-316.

4 Chen YK, Huang HC, Lin LM et al. Primary oral squamous cell carcinoma: an analysis of 703 cases in southern Taiwan. Oral Oncol 1999; 35(2): 173-179.

5 Zhou ZT, Jiang WW. Cancer stem cell model in oral squamous cell carcinoma. Curr Stem Cell Res Ther 2008; 3(1): 17-20.

6 Costea DE, Tsinkalovsky O, Vintermyr OK et al. Cancer stem cells-new and potentially important targets for the therapy of oral squamous cell carcinoma. Oral Dis 2006; 12(5): 443-454.

7 Felthaus 0 , Ettl T, Gosau M et al. Cancer stem cell-like cells from a single cell of oral squamous carcinoma cell lines. Biochem Biophys Res Commun 2011; 407(1): 28-33. 
8 Oliveira LR, Oliveira-Costa JP, Araujo IM et al. Cancer stem cell immunophenotypes in oral squamous cell carcinoma. J Oral Pathol Med 2011; 40(2): 135-142.

9 Chen YK, Lin LM. DMBA-induced hamster buccal pouch carcinoma and VX2-induced rabbit cancer as a model for human oral carcinogenesis. Expert Rev Anticancer Ther 2010; 10(9): 1485-1496.

10 Lin LM, Chen YK, Chen CH et al. VX2-induced rabbit buccal carcinoma: a potential cancer model for human buccal mucosa squamous cell carcinoma. Oral Oncol 2009; 45(11): e196-e203.

11 Singh SK, Clarke ID, Terasaki $M$ et al. Identification of a cancer stem cell in human brain tumors. Cancer Res 2003; 63(18): 5821-5828.

12 Zhang S, Balch C, Chan MW et al. Identification and characterization of ovarian cancer-initiating cells from primary human tumors. Cancer Res 2008; 68(11): 4311-4320.

13 Qiu X, Wang Z, Li Y et al. Characterization of sphere-forming cells with stem-like properties from the small cell lung cancer cell line H446. Cancer Lett 2012; 323(2): 161-170.

14 Lim YC, Oh SY, Cha YY et al. Cancer stem cell traits in squamospheres derived from primary head and neck squamous cell carcinomas. Oral Oncol 2011; 47(2): 83-91.

15 Cicalese A, Bonizzi G, Pasi CE et al. The tumor suppressor p53 regulates polarity of self-renewing divisions in mammary stem cells. Cell 2009; 138(6): 1083-1095.

16 Pastrana E, Silva-Vargas V, Doetsch F. Eyes wide open: a critical review of sphereformation as an assay for stem cells. Cell Stem Cell 2011; 8(5): 486-498.

17 Prince ME, Sivanandan R, Kaczorowski A et al. Identification of a subpopulation of cells with cancer stem cell properties in head and neck squamous cell carcinoma. Proc Natl Acad Sci U S A 2007; 104(3): 973-978

18 Pries R, Witrkopf N, Trenkle T et al. Potential stem cell marker CD44 is constitutively expressed in permanent cell lines of head and neck cancer. In Vivo 2008; 22(1): 8992.

19 Oliveira LR, Oliveira-Costa JP, Araujo IM et al. Cancer stem cell immunophenotypes in oral squamous cell carcinoma. J Oral Pathol Med 2011; 40(2): 135-142.

20 Marcato P, Dean CA, Giacomantonio CA et al. Aldehyde dehydrogenase: its role as a cancer stem cell marker comes down to the specific isoform. Cell Cycle 2011; 10(9): 1378-1384.

21 Chute JP, Muramoto GG, Whitesides J et al. Inhibition of aldehyde dehydrogenase and retinoid signaling induces the expansion of human hematopoietic stem cells. Proc Natl Acad Sci U S A 2006; 103(31): 11707-11712.
22 Chen YC, Chen YW, Hsu HS et al. Aldehyde dehydrogenase 1 is a putative marker for cancer stem cells in head and neck squamous cancer. Biochem Biophys Res Commun 2009; 385(3): 307-313.

23 Krishnamurthy S, Dong Z, Vodopyanov D et al. Endothelial cell-initiated signaling promotes the survival and self-renewal of cancer stem cells. Cancer Res 2010; 70(23): 9969-9978.

24 Yin AH, Miraglia S, Zanjani ED et al. AC133, a novel marker for human hematopoietic stem and progenitor cells. Blood 1997; 90(12): 5002-5012.

25 Miraglia S, Godfrey W, Yin AH et al. A novel five-transmembrane hematopoietic stem cell antigen: isolation, characterization, and molecular cloning. Blood 1997; 90(12): 5013-5021.

26 Zhang Q, Shi S, Yen Y et al. A subpopulation of $\mathrm{CD} 133^{+}$cancer stem-like cells characterized in human oral squamous cell carcinoma confer resistance to chemotherapy. Cancer Lett 2010; 289(2): 151-160.

27 Park IK, Morrison SJ, Clarke MF. Bmi1, stem cells, and senescence regulation. J Clin Invest 2004; 113(2): 175-179.

28 Park IK, Qian D, Kiel M et al. Bmi-1 is required for maintenance of adult self-renewing haematopoietic stem cells. Nature 2003; 423(6937): 302-305.

29 Molofsky AV, Pardal R, Iwashita T et al. Bmi-1 dependence distinguishes neural stem cell self-renewal from progenitor proliferation. Nature 2003; 425(6961): 962-967.

30 Chen YC, Chang $\mathrm{CJ}$, Hsu HS et al. Inhibition of tumorigenicity and enhancement of radiochemosensitivity in head and neck squamous cell cancer-derived ALDH1 positive cells by knockdown of Bmi-1. Oral Oncol 2010; 46(3): 158-165.

31 Zhang Z, Filho MS, Nör JE. The biology of head and neck cancer stem cells. Oral Onco 2012; 48(1): 1-9.

32 Cheng L, Alexander R, Zhang S et al. The clinical and therapeutic implications of cancer stem cell biology. Expert Rev Anticancer Ther 2011; 11(7): 1131-1143.

cc)(i)(2) This work is licensed under a Creative Commons AttributionNonCommercial-ShareAlike 3.0 Unported License. The images or other third party material in this article are included in the article's Creative Commons license, unless indicated otherwise in the credit line; if the material is not included under the Creative Commons license, users will need to obtain permission from the license holder to reproduce the material. To view a copy of this license, visit http://creativecommons.org/licenses/by-nc-sa/3.0/ 